\title{
Sustainable Information Systems: a knowledge perspective
}

\author{
Laura Măruşter, Niels R. Faber, and Kristian Peters \\ University of Groningen, Faculty of Economics and Business, \\ PO Box 800, 9700 AV Groningen, the Netherlands \\ \{1.maruster, n.r.faber, k. peters\}@rug.nl
}

\begin{abstract}
We propose a reorientation of the way the concept of sustainability is dealt with in relation to information systems, positioning the processing of knowledge at the centre of the concept. The concept of Sustainability of Knowledge (SoK), referring to processes that govern knowledge is employed to define Sustainable Information Systems (SIS). Three knowledge aspects are found to be relevant for the design of Sustainable Information Systems: adaptability, offloading and knowledge evaluation. The proposed sustainability approach is translated into requirements needed for SIS, by employing a SOA architecture.
\end{abstract}

Key words: sustainability, knowledge management, adaptation, offloading, knowledge evaluation, stakeholders, SOA

\section{Introduction}

Current literature acknowledges that sustainability is a broad, complex concept [1], involving environmental as well as social issues, and which requires continuous learning in order to be understood and tackled. However, the issue of sustainability is still mostly connected to ecological and environmental terms.

The discussion about sustainability and Information Systems (ISs) appears in different contexts. For instance, models and tools have been developed to assess corporate sustainability [2] and sustainability of Management Information Systems [3]. Often, the notion of sustainability of ISs stems from the broader notion of Sustainable Development and is applied in a specific domain. Also, contributions concerning sustainability and Information Systems originate from joining domains, such as sustainability and systems [4].

This article proposes a reorientation of the way the concept of sustainability is dealt with, positioning human behaviour and the processing of knowledge at the centre of the concept. The sustainability approach presented in this paper is then translated into requirements needed for designing an Sustainable Information System (SIS).

Section 2 sets out our position from a social perspective, where we use the notions of knowledge, adaptability and offloading. Section 3 discusses the requirements of an IS that conforms with our notion of sustainability, centred around knowledge aspects. In section 4 we present our conclusions and further research. 


\section{The social perspective on sustainability}

The relation between human behaviour and issues of sustainability is conceptualised using the notion of artificial system [5]. An artificial system is defined as a system that is (i) made by humans and (ii) is operated by humans [1]. Therefore, an IS is treated as an artificial system, because (i) IS's are human-made, and (ii) IS's are operated by humans. From the definition of the artificial system and given that human actions follow from an individual's knowledge, knowledge is identified to control the artificial system.

The first notion used to approach sustainability is knowledge. Three related terms are placed in the sequence data - information - knowledge. Data concerns the signals that humans receive using their senses. One level higher, data is used to form information, which concerns the interpretation of data. Finally, knowledge is interpreted information, which enables humans to apply the information in reasoning, decision-making, or performing actions. Knowledge used to operate an IS needs to be updated continuously. Individuals, who control the IS, have to cope with the changes of the system to maintain an equilibrium between the system and its environment. We call this Sustainability of Knowledge (SoK), which means that all knowledge processes need to be guided to lead to the development of new knowledge. Three criteria need to be met by ISs, in order to establish an appropriate balance of all knowledge processes leading to SoK. An IS should (a) allow the creation of knowledge, (b) enable the critical evaluation of knowledge, and (c) ensure the effective integration and application of knowledge.

The second notion used for sustainability is adaptation. Adaptation means that an organization needs to ensure that its interactions with its environment fit the demands and possibilities of this environment. In some way, the organization's functions need to be aligned with in- and outputs that the environment provides or allows. Organizational functions are realized by humans in processes and tasks, thereby supported by machines and all sorts of information systems. An alignment of organizational functions implies the alteration, reorganization, and redistribution of the organizations processes and tasks.

The third notion used is offloading. Offloading involves burdening, harming, destroying or exploiting the economic, ecological and/or social aspects of the environment [1]. When a unbalance exists between the environment and an organisation and its supporting IS's, sustainability can be reached by attempting to achieve a reduction in offloading, by involving stakeholders. Stakeholders can be involved in the organizational sense-making, strategy-forming, and decisionmaking processes in order to answer the questions how they suffer from the firm's offloading and to what extend. Stakeholders are "those groups and individuals who can affect, or are affected by the achievement of an organization's purpose" [6]. Regarding IS's within organizations, two groups of stakeholders are identified: (a) stakeholders related to the business system (employees, legislators), and (b) IS stakeholders, consisting for instance IS-developers or programmers. The employees of the organization who use the IS also are considered part of the IS stakeholders. 


\section{Requirements for designing an SIS}

Summing up, a Sustainable Information System (SIS) is an Information System which (i) adapts to its environment, (ii) involves relevant stakeholders, and (iii) supports the knowledge lifecyle, i.e. knowledge creation, knowledge evaluation and knowledge integration/application.

We illustrate SIS design requirements by using a platform that seems suitable to support our approach, namely Service Oriented-based Architecture [7]. Figure 1 shows our SOA-based solution, consisting of four separate layers: the Business Process layer, the Application layer, the Service layer and what we call the Knowledge layer. The first three layers are the standard layers of a SOAbased architecture. The rational of the additional Knowledge layer is to address specific knowledge aspects. Concerning adaptability, SIS (i) should be equipped with mechanisms that detect and deal with changes occurring between IS and its environment, (ii) the IS forms a suitable platform for dealing with changes. The theory of Complex Adaptive Systems (CAS) seems to provide a suitable framework for dealing with change [8]. CAS theory consider that systems (for instance organizations made up of human and software agents) self-organize and adapt to their changing environment. Offloading can be dealt with through additional services that manage stakeholder inputs. Such services can be an error-reporting service, which enables stakeholders to report errors they encounter while using the information system, or a survey service that regularly questions the stakeholders about the functionality and useability that is offered by the information system.

Figure 1 shows our SOA-based solution to support the proposed SIS approach. Concerning adaptability and offloading concerns, the SOA paradigm is an option for addressing adaptation and offloading problems, because it provides solutions for enterprise-wide loose coupling, support for service-oriented business modelling, organisational agility and layers of abstraction [7].

The architecture proposed in figure 1 provides several advantages concerning adaptation and offloading. First, it allows the decoupling between different layers: changes occurring in a certain layer are easily mastered within that layer, by means of the Orchestration Service layer. Second, by incorporating in the Knowledge layer current organizational knowledge, it allows the detection of the discrepancies between the (knowledge about) environment and information system, and enable the solution search.

\section{Conclusions and further research}

We propose a reorientation of the way the concept of sustainability is dealt with, positioning knowledge issues at the centre of the concept. The notion of sustainability in relation to knowledge is employed to define SIS: Sustainability of Knowledge (SoK), which refers to processes governing knowledge. Three knowledge aspects are relevant regarding SISs: adaptability, offloading and knowledge lifecycle. We translate these aspects into requirements needed for designing a SIS, 


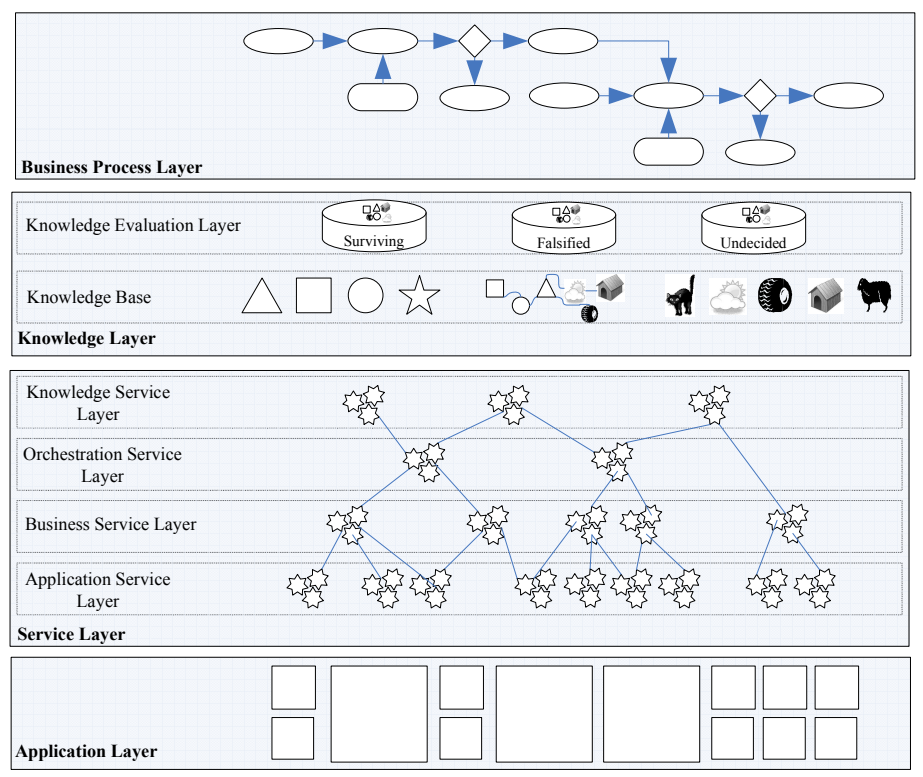

Fig. 1. SIS proposed architecture

by employing a Service Oriented-based Architecture. An additional Knowledge layer is added, consisting of a Knowledge Base and a Knowledge Evaluation layer. The function of this Knowledge layer is to support all three knowledge aspects relevant to sustainability. As further research, we aim to perform case studies in different organizations that aim to test the proposed approach.

\section{References}

1. Faber, N.R., Jorna, R.J., van Engelen, J.M.: The sustainability of "sustainability": a study into the conceptual foundations of the notion of "sustainability". Journal of Environmental Assessment Policy and Management, 7(1), 1-33 (2005)

2. Zwetsloot, G.I., van Marrewijk, M.N.: From Quality to Sustanability. J Bus Ethics, 55, 79-82 (2004)

3. Caldelli, A., Parmigiani, M.L.: Management information system - A tool for corporate sustainability. J Bus Ethics, 55, 159-171 (2004)

4. Drinan, J. In Frank A. Stowell, Ray L. Ison, e.a., ed.: Systems for Sustainability. People, Organizations and Environments. Plenum Press (1997)

5. Simon, H.A.: The sciences of the artificial. The MIT Press, Cambridge, Massachusetts (1969)

6. Amaeshi, K.M., Crane, A.: Stakeholder engagement: A mechanism for sustainable aviation. C.S.R. \& E.M. 13, 245-260 (2006)

7. Erl, T.: Service-Oriented Architecture: Concepts, Technology, and Design. Prentice Hall PTR (2005)

8. Holland, J.: Hidden Order: How Adaptation Builds Complexity. Reading, MA: Perseus Books (1995) 